\title{
Best Error Bounds of Quartic Spline Interpolation
}

\author{
Y.P.Dubey ${ }^{1}$ \\ L.N.C.T.ENG.COLLEGE JBP
}

\author{
K.K.Nigam ${ }^{2}$ \\ L.N.C.T.ENG. COLLEGE JBP
}

\begin{abstract}
In this paper, we have obtained existence, uniqueness, best error bound and convergence properties of $\mathrm{C}^{2}$ Deficient Quartic Spline Interpolation.
\end{abstract}

\section{Classification Code}

Ø 41A05, 65D07.

\section{INTRODUCTION}

Higher Degree Splines are still popular for best approximation (See DeBoor [1]). In the study of higher degree piecewise spline function it advantage that we do not have corners at joint of two higher degree pieces and therefore to achieve a prescribed accuracy less data than lower order method needed. These types of advantage do not appear in the use of piecewise linear splines (lower order splines). Rana and Dubey [8] generalized result of Garry and Howell [3] and obtained best error bound for quartic spline interpolation. Various aspects of cubic, quartic, quintic and spline of degree six reference maya be given Meir and Sharma [9], Hall and Mayer [2], Gemlling-Meyling [6], Rana and Dubey [7], and Dubey [5]. In this paper we have investigated existence uniqueness, error bounds and convergence properties of deficient quartic spline interpolation which matches the given function of mesh points and mid points, second derivative at boundary points.

\section{EXISTENCE AND UNIQUENESS}

Consider a mesh $\mathrm{P}$ of $[0,1]$ given by $0=x_{0}<x_{1}<,<x_{n}=1$ such that $x_{i+1}-x_{i}=h_{i} \mathrm{i}=0$, 1.......n-1. Let $\mathrm{S}(4, \mathrm{P})$ denote the set of all real algebric polynomial of degree not greater than 4 . We denote $s_{i}$ is the restriction of $\mathrm{s}$ over $\left[x_{i}, x_{i+1}\right]$ for a function defined over $[0,1]$. The class of deficient quartic spline functions over $[0,1]$ with mesh $\mathrm{P}$ is defined by

$S(4, P)=\left[\left\{s: s \in C^{2}[0,1], s_{i} \in S(4, P), i=1,2, . ., n-1\right\}\right]$

which is $\mathrm{S}^{*}(4, \mathrm{P})$ denotes the class of all deficient quartic splines $\mathrm{S}(4, \mathrm{P})$ which satisfy the boundary conditions

$$
\begin{aligned}
& s^{\prime \prime}\left(x_{0}\right)=f^{n}\left(x_{0}\right) \\
& s^{\prime \prime}\left(x_{n}\right)=f^{n}\left(x_{n}\right)
\end{aligned}
$$

We shall prove the following.
THEOREM 2.1: Let $f^{\prime}, f^{\prime \prime}$ exist, then there exist a unique deficient quartic spline in $\mathrm{S}^{*}(4, \mathrm{P})$ which satisfies the interpolatory conditions.

$$
\begin{aligned}
& s\left(x_{1}\right)=f\left(x_{i}\right) i=0,1, \ldots n \\
& s\left(z_{i}\right)=f\left(z_{i}\right) i=0,1, \ldots n-1
\end{aligned}
$$

PROOF: Consider a quartic polynomial P $(\mathrm{z})$ on $[0,1]$, we can easily verify that

$$
\begin{aligned}
& P(z)=P(0) q_{1}(z)+P(1) q_{2}(z)+P(1 / 2) q_{3}(z) \\
& +P^{\prime \prime}(0) \cdot q_{4}(z)+P^{\prime \prime}(1) q_{5}(z)
\end{aligned}
$$

Where,

$$
\begin{aligned}
& q_{1}(z)=\frac{1}{30}\left[30-78 z+96 z^{3}-48 z^{4}\right] \\
& q_{2}(z)=\frac{1}{30}\left[-18 z+96 z^{3}-48 z^{4}\right] \\
& q_{3}(z)=\frac{1}{30}\left[96 z-192 z^{3}+96 z^{4}\right] \\
& q_{4}(z)=\frac{1}{30}\left[-4 z+15 z^{2}-17 z^{3}+6 z^{4}\right] \\
& q_{5}(z)=\frac{1}{30}\left[z-7 z^{3}+6 z^{4}\right]
\end{aligned}
$$

Now writing $h_{i} t=\left(x-x_{i}\right)$ (2.4) may be expressed in terms of restriction $S_{i}$ of s as follows

$$
\begin{aligned}
s_{i}(x)= & f\left(x_{i}\right) q_{1}(t)+f\left(x_{i+1}\right) q_{2}(t)+f\left(z_{i}\right) q_{3}(t) \\
& +s^{\prime \prime}\left(x_{i}\right) q_{4}(t)+s^{\prime \prime}\left(x_{i+1}\right) q_{5}(t)
\end{aligned}
$$

Which clearly satisfies the conditions (2.1) - (2.3) and

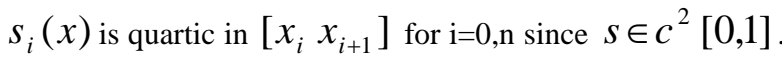
Therefore, applying continuity conditions of first derivative of $\mathrm{s}$ in (2.5) we have 
$h_{i-1} s^{\prime \prime}\left(x_{i-1}\right)-4 s^{\prime \prime}\left(x_{i}\right)\left(h_{i-1}+h_{i}\right)+h_{i} s^{\prime \prime}\left(x_{i+1}\right)=F_{i}$

Where $F_{i}=18\left[\frac{f\left(x_{i-1}\right)}{h_{i-1}}+\frac{f\left(x_{i}\right)}{h_{i}}\right]$

$+78 f\left(x_{i}\right)\left(\frac{1}{h_{i-1}}+\frac{1}{h_{i}}\right)-96\left(\frac{f\left(z_{i-1}\right)}{h_{i-1}}+\frac{f\left(z_{i}\right)}{h_{i}}\right)$

Clearly the coefficient matrix of the system of equation (2.6) is diagonally dominant and hence invertible. This complete proof of theorem (2.1).

\section{ERROR BOUNDS}

Following the method of Hall and Meyer [2] in this section, we shall obtain bounds of error function, $e(x)=f(x)-s(x)$ for the spline interpolant of Theorem 2.1 which are best possible. Let $\mathrm{s}(\mathrm{x})$ is the second time continuously differentiable quartic spline function satisfy the condition of theorem 2.1. Now considering $f \in C^{5}[0,1]$ and writing $M_{i}[f, x]$ for the unique quartic which agree with $f\left(x_{i}\right), f\left(x_{i-1}\right), f\left(z_{i}\right), f^{\prime \prime}\left(x_{i}\right)$ and $f^{\prime \prime}\left(x_{i+1}\right)$ we see that for $x \in\left[x_{i}, x_{i+1}\right]$ we have

$$
\begin{aligned}
& |f(x)-s(x)| \cong\left|f(x)-s_{1}(x)\right| \\
& \leq\left|f(x)-M_{i}[f, x]\right|+\left|M_{i}[f, x]-s_{i}(x)\right|
\end{aligned}
$$

First, we have to obtain bounds of right hand side of

By Cauchy theorem Davis [4], we have obtain

$$
\left|f(n)-h_{i}[f, x]\right| \leq \frac{h^{5}}{5 !}\left|t^{2}(1-t)^{2}\left(t-\frac{1}{2}\right)\right|
$$

Where $t=\frac{x-x_{i}}{h_{i}}$ and $F=\max _{0 \leq n \leq 1}\left|f^{(5)}(x)\right|$

To get the bounds of $\left|M_{i}[f, x]-s_{i}(x)\right|$

We have from (2.4)

$$
\begin{aligned}
& M_{i}[f, x]-s_{i}(x)=h_{i}^{2}\left[f^{\prime \prime}\left(x_{i-1}\right)-s^{\prime \prime}\left(x_{i-1}\right)\right] \\
& q_{4}(t)+h_{i}^{2}\left[f^{\prime \prime}\left(x_{i}\right)-s^{\prime \prime}\left(x_{i}\right)\right] q_{5}(t)
\end{aligned}
$$

Thus

$$
\begin{aligned}
& \left|M_{i}[f, x]-s_{i}(x)\right| \\
& \leq\left|h_{i-1}^{2} e^{\prime \prime}\left(x_{i-1}\right) q_{4}(t)\right|+h_{i}^{2}\left|e^{\prime \prime}\left(x_{i}\right) q_{5}(t)\right|
\end{aligned}
$$

$\left|q_{4}(t)+q_{5}(t)\right| \leq\left|q_{4}(t)\right|+\left|q_{5}(t)\right|$

$=\frac{1}{6}(1-t)(1-2 t)=k_{1}(t)$ Say when $0 \leq t \leq \frac{1}{2}$

$=\frac{1}{6} t(1-t)(2 t-1)=k_{2}(t)$ Say

When $\frac{1}{2} \leq t \leq 1$

Let $k(t)=\max _{0 \leq t \leq 1}\left\{k_{1}(t), k_{2}(t)\right\}$

Let the $\max _{0 \leq t \leq 1}\left|e\left(x_{i}\right)\right|$ exists for $\mathrm{i}=\mathrm{j}$ then the equation (3.5) may be written as

$$
\left|M_{i}[f, x]-s_{i}(x)\right| \leq h_{1}^{2}\left|e^{\prime \prime}\left(x_{i}\right)\right| k(t)
$$

Now, we proceed to obtain $\left|e\left(x_{i}\right)\right|$ replacing $s\left(x_{i}\right)$ by $e\left(x_{i}\right)$ in equation (2.6), we have

$$
\begin{aligned}
& h_{i-1} e_{i-1}^{11}-4 e_{i}^{11}\left(h_{i-1}+h_{i}\right)+h_{i} e_{i+1}^{11} \\
& =F_{i}-h_{i-1} f_{i-1}^{11}+4 f_{1}^{11}\left(h_{i-1}+h_{i}\right)-h_{i} f_{i+1}^{11}=L(f)
\end{aligned}
$$

In view of that $L(f)$ is linear functional which is zero for polynomials of degree 4 or less, we can apply the Peano Theorem Davis [4] to obtain

$$
L(f)=\int_{x_{i-1}}^{x_{i+1}} \frac{f^{(5)}(y)}{4 !} L\left[(x-y)_{+}^{4}\right] d y
$$

Thus, from (3.8), we have

$$
|L(f)| \leq \frac{1}{4 !} F \int_{x_{i-1}}^{x_{i+1}}\left|L(x-y)_{+}^{4}\right| d y
$$

Further it can be observed that from (3.9) that in $x_{i-1} \leq x \leq x_{j+1}$

$$
\begin{aligned}
& L\left[\left(x_{i}-y\right)_{+}^{4}\right]=\frac{18}{h_{i}}\left(x_{i+1}-y\right)^{4} \\
& +78\left(\frac{1}{h_{i-1}}+\frac{1}{h_{i}}\right)\left(x_{i}-y\right)_{+}^{4} \\
& -\frac{96}{h_{i-1}}\left(z_{i-1}-y\right)_{+}^{4}-\frac{96}{h_{i}}\left(z_{i}-y\right)_{+}^{4}+48\left(x_{i}-y\right)_{+}^{2} \\
& \left(h_{i-1}+h_{i}\right)-12 h_{i}\left(x_{i+1}-y\right)^{2}
\end{aligned}
$$

Thus we have 
In order to estimate the interval of r.h.s. of (3.10), we rewrite the above expression in the following symmetric form about $\mathrm{x}_{\mathrm{i}}$, to get

$$
\begin{gathered}
\Rightarrow \frac{6}{h_{i}}\left(x_{i}-y+h_{i}\right)^{2}\left[-2 h_{i}^{2}+3\left(x_{i}-y+h_{i}\right)^{2}\right] \\
z_{i} \leq y \leq x_{i+1}
\end{gathered}
$$$$
\Rightarrow-\frac{6}{h_{i}}\left[13\left(x_{i}-y\right)^{2}+20 h_{i}\left(x_{i}-y\right)^{2}+8 h_{i}^{2}\right]\left(x_{i}-y\right)^{2}
$$$$
x_{i} \leq y \leq z_{i}
$$$$
\Rightarrow \frac{6}{h_{i-1}}\left[13\left(x_{i}-y\right)^{2}-20 h_{i-1}\left(x_{i}-y\right)+8 h_{i-1}^{2}\right]\left(x_{i}-y\right)^{2}
$$

$$
z_{i-1} \leq y x_{i}
$$$$
\Rightarrow-\frac{6}{h_{i-1}}\left(x_{i}-y+h_{i-1}\right)^{2}\left[-2 h_{i-1}^{2}+3\left(x_{i}-y-h_{i-1}\right)^{2}\right]
$$

$$
x_{i-1} \leq y z_{i-1}
$$

$$
\left|e^{11}(x)\right| \leq K_{1}\left(\frac{h^{3}}{5 !}\right) \max _{0 \leq x \leq 1}\left|f^{5}(x)\right|
$$

Thus its clear from above expression that $L\left[(x-y)_{+}^{4}\right]$ is non negative for $x_{i-1} \leq y \leq x_{i+1}$

$$
\int_{n_{i-1}}^{n_{i+1}}\left|L(x-y)_{+}^{4}\right| d y=\frac{93}{80}\left(h_{i-1}^{4}+h_{i}^{4}\right)
$$

Combining (3.10) and (3.11), we have

$$
|L(f)| \leq \frac{93 F}{16 \times 5 !}\left(h_{i-1}^{4}+h_{i}^{4}\right)
$$

Thus from (3.8) and (3.11)

$$
\left|e^{11}\left(x_{i}\right)\right| \leq\left|e_{i}^{11}\right| \leq \frac{31 F}{16(5 !)}\left(\frac{h_{i-1}^{4}+h_{i}^{4}}{h_{i-1}+h_{i}}\right)
$$

Now, using (3.2), (3.7) along with (3.13) in (3.1), we have

$$
\begin{aligned}
& |e(x)| \leq \frac{h^{5}}{5} \mid t^{2}(1-t)^{2}\left(t-\frac{1}{2}\left|F+h_{i}^{2}\right| e^{11}\left(x_{i}\right) \mid k(t)\right. \\
& =\frac{h^{5}}{5 !}\left|t^{2}(1-t)^{2}\left(t-\frac{1}{2}\right)\right| F+\frac{F h^{5}}{5 !} \frac{(31)}{(16)} k(t)
\end{aligned}
$$

$\leq \frac{h^{5} F}{5 !} \mid c(t)$

Where $c(t)=\left[t^{2}(1-t)^{2}\left(t-\frac{1}{2}\right)+\frac{31}{16} k(t)\right]$
Thus we prove the following:

THEOREM 3.1: Suppose $s(x)$ is the quartic spline of Theorem $2.1 \quad$ interpolating a function $f(x)$ and $f \in C^{5}[0,1]$, then

$$
|e(x)| \leq K \frac{h^{5}}{5 !} \max _{0 \leq t \leq 1}\left|f^{(5)}(x)\right|
$$

Where $K=\max _{0 \leq t \leq 1}|c(t)|$

$$
\begin{aligned}
& =\max _{0 \leq t \leq 1}\left[t^{2}(1-t)^{2}\left(t-\frac{1}{2}\right)+\frac{31}{16} k(t)\right] \text { And } \\
& \left|e^{11}\left(x_{i}\right)\right| \leq \frac{1}{5 !} \frac{31}{(16)} h^{3} \max _{0 \leq t \leq 1}\left|f^{(5)}(x)\right|
\end{aligned}
$$

Further more, it can be seen easily that $\mathrm{K}$ in (3.17) be improved for an equally spaced partition. Inequality (3.18) is also best possible. Also, we have

Where $K_{1}$ is positive constant Equation (3.14) proves inequality (3.17) whereas inequality (3.18) is direct consequence of (3.13).

Now we turn to see that the inequality (3.17) is best possible in the limit. Considering $f(x)=\frac{x^{5}}{5 !}$ and using Cauchy formula Davis [4] we have

$M_{i}\left[\frac{x^{5}}{5 !}, x\right]-\frac{x^{5}}{5 !}=h^{5}(1-t)^{2} t^{2}\left(t-\frac{1}{2}\right) / 5 !$

Moreover, for the function under consideration (3.7) the following relation holds for equally spaced knots

$M_{i}\left[\frac{x^{5}}{5 !}\right]=\frac{31}{16(5 !)} h^{3}=e_{j-1}^{11}-8 e_{j}^{11}+e_{j+1}^{11}$

Considering for a moment

$$
e_{j}^{11}=-\frac{31}{96} \frac{h^{3}}{(5 !)}=e_{j-1}^{11}=e_{j+1}^{11}
$$

We have from (3.4)

$s(n)-M_{i}[f, x]=-\frac{31 h^{5}}{96(5 !)}\left(q_{4}(t)+q_{5}(t)\right)$ 
$=+\frac{31}{96} \frac{h^{5}}{(5 !)}\left[t(1-t)(1-2 t)^{2}\right]$

Now combining (3.20) and (3.23) we get, for $x_{i} \leq x \leq x_{i+1}$

$s(n)-f(n)=\frac{h^{5}}{5 !}\left\{\frac{31}{96}(1-2 t)+\frac{t(1-t)}{2}\right\} t(1-t)(1-2 t)$ Now again using (3.26) in (3.21) we have

$$
8 e_{j}^{11}=-\frac{31 h^{3}}{16(5 !)}+e_{j-i}^{11}+e_{j+1}^{11}
$$

$\Rightarrow e_{j}^{11} \leq \frac{1}{8}\left[-\frac{31 h^{3}}{16(5 !)}\right]$

From (3.24) it is clearly observed that (3.17) is best possible, provided we could prove that

$e_{i-1}^{11}=e_{i}^{11}=e_{i+1}^{11}=-\frac{31 h^{3}}{96(5 !)}$

In fact (3.25) is attained only in the limit, the difficulty will appear in the case of boundary conditions i.e. $e^{11}\left(x_{0}\right)=e^{11}\left(x_{n}\right)=0$. However, it can be shown that as we were many subintervals away from the boundaries $e^{11}\left(x_{i}\right) \rightarrow-\frac{31}{96} \frac{h^{3}}{(5 !)}$. For that, we shall apply (3.20) inductively to move away from the end conditions $e^{11}\left(x_{0}\right)=e^{11}\left(x_{n}\right)=0$.

The first step in this direction is to establish that $e^{11}\left(x_{i}\right) \leq 0$ for some $\mathrm{i}, \mathrm{i}=1,2, \ldots . \mathrm{n}$, which can be shown by contradictory result. Let $e^{11}\left(x_{i}\right)>0$ for some $\mathrm{i}=1,2, \ldots \mathrm{n}-1$. Now making use of (3.18), we get

$$
\frac{31}{96} \frac{h^{3}}{(5 !)} \geq \max _{0 \leq x \leq 1}\left|e^{11}\left(x_{j}\right)\right| \geq \frac{1}{2}\left[e_{j-1}^{11}+e_{j+1}^{11}\right]
$$

$$
\begin{aligned}
>\frac{1}{2}\left[e_{j-1}^{11}-8 e_{j+1}\right. & \left.+e_{j+1}^{11}\right] \\
& >\frac{1}{2}\left[\frac{31 h^{3}}{16(5 !)}\right]
\end{aligned}
$$$$
1>3
$$

This is the contradiction, hence

$$
e^{11}\left(x_{j}\right) \leq 0
$$

$$
\Rightarrow e^{11}\left(x_{j}\right) \leq-\frac{31 h^{3}}{(5 !) 16 \times 8}\left[1+\frac{2}{8}\right]
$$

Repeated use of (3.21) follows that

$$
e^{11}\left(x_{j}\right) \leq-\frac{31 h^{3}}{(5 !) 16 \times 8}\left[1+\frac{2}{8}+\left(\frac{2}{8}\right)^{2}+\ldots \ldots . .\right]
$$

Now it can be seen easily that right hand side of (3.28) $\rightarrow \frac{-31 h^{3}}{96(5 !)}$ and hence in the limiting case $e^{11}\left(x_{j}\right) \leq-\frac{31 h^{3}}{96(5 !)}$ which verify proof the inequality (3.19).

Thus corresponding to the function $f(x)=\frac{x^{5}}{5 !}$, (3.27) and (3.28) tends to $-\frac{31}{96} \frac{h^{3}}{(5 !)}$ in the limit for equally spaced knots. This complete proof of theorem 3.1.

\section{REFERENCES}

[1] Deboor, C.A. Practical Guide to Splines, Applied Mathematical Science, Vol. 27 Spoinger, Varlag, New York 1979.

[2] Hall, C.A. and Meyer, W.W., J. Approximation Theory 16 (1976), pp 105-122.

[3] Howell, G. and Verma, A.K. Best Error Bound of Quartic Spline Interpolation, J. Approx. Theory 58 (1989), 58-67.

[4] Davis, P.J. Interpolation and approximation, New York, 1969.

[5] Dubey, Y.P. Best Error Bounds of Spline of degree six. Int.Jour. of Mathematical Ana. Vol. 5 (2011), pp. 21-24.

[6] Gemlling, R.H.J. and Meyling, G. in Interpolation by Bivartate Quintic Splines of Class Construction of Theory of function 87 (ed) Sender et al (1987) 152-61.

[7] Rana, S.S. and Dubey, Y.P. Best ERror Bounds of Quintic Spline Interpolation J. Pune and App. Math 28 (10) 1937-44 (1997). 
[8] Rana, S.S. and Dubey, Y.P. Best Error Bounds of deficient quartic spline interpolation, Indian Journal Pune and Appl. Math 30(4) (1999), 385-393.
[9] Meir, A. and Sharma, A. Convergence of a class of interpolatory spline J. Approx. Theory (1968), pp. 243250 . 